\title{
Magnetic-buoyancy-induced mixing in AGB stars: Fluorine nucleosynthesis at different metallicities
}

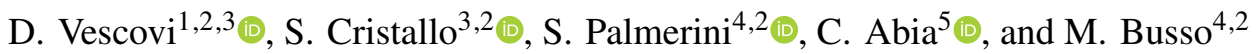 \\ ${ }^{1}$ Goethe University Frankfurt, Max-von-Laue-Strasse 1, Frankfurt am Main 60438, Germany \\ e-mail: vescovi@iap.uni-frankfurt.de \\ 2 INFN, Section of Perugia, Via A. Pascoli snc, 06123 Perugia, Italy \\ 3 INAF, Observatory of Abruzzo, Via Mentore Maggini snc, 64100 Teramo, Italy \\ ${ }^{4}$ Department of Physics and Geology, University of Perugia, Via A. Pascoli snc, 06123 Perugia, Italy \\ ${ }^{5}$ University of Granada, Departamento de Fisica Teorica y del Cosmos, 18071 Granada, Spain
}

Received 23 April 2021 / Accepted 15 June 2021

\begin{abstract}
Asymptotic giant branch (AGB) stars are considered to be among the most significant contributors to the fluorine budget in our Galaxy. While observations and theory agree at close-to-solar metallicity, stellar models at lower metallicities overestimate the fluorine production with respect to that of heavy elements. We present ${ }^{19} \mathrm{~F}$ nucleosynthesis results for a set of AGB models with different masses and metallicities in which magnetic buoyancy acts as the driving process for the formation of the ${ }^{13} \mathrm{C}$ neutron source (the so-called ${ }^{13} \mathrm{C}$ pocket). We find that ${ }^{19} \mathrm{~F}$ is mainly produced as a result of nucleosynthesis involving secondary ${ }^{14} \mathrm{~N}$ during convective thermal pulses, with a negligible contribution from the ${ }^{14} \mathrm{~N}$ present in the ${ }^{13} \mathrm{C}$ pocket region. A large ${ }^{19} \mathrm{~F}$ production is thus prevented, resulting in lower fluorine surface abundances. As a consequence, AGB stellar models with mixing induced by magnetic buoyancy at the base of the convective envelope agree well with available fluorine spectroscopic measurements at low and close-to-solar metallicity.
\end{abstract}

Key words. stars: abundances - stars: AGB and post-AGB - stars: magnetic field - magnetohydrodynamics (MHD) - stars: carbon nuclear reactions, nucleosynthesis, abundances

\section{Introduction}

Understanding the cosmic origin of fluorine is one of the most interesting topics in the field of nuclear astrophysics. The production of its sole stable isotope, ${ }^{19} \mathrm{~F}$, largely depends on the physical conditions characterizing stellar interiors. The primary source of fluorine in the Universe is currently widely debated, and several sites have been proposed as potential candidates: asymptotic gaint branch (AGB) stars (Forestini et al. 1992), rapidly rotating massive stars (Limongi \& Chieffi 2018), WolfRayet stars (Meynet \& Arnould 2000), core-collapse supernovae (Woosley \& Haxton 1988), and novae (José \& Hernanz 1998). While the contributions from many of these sources are required to explain the galactic chemical evolution of fluorine abundance (see, e.g., Meynet \& Arnould 2000; Renda et al. 2004; Kobayashi et al. 2011; Spitoni et al. 2018; Prantzos et al. 2018; Olive \& Vangioni 2019; Grisoni et al. 2020), the only direct observation of fluorine production is provided by spectroscopic findings of photospheric $[\mathrm{F} / \mathrm{Fe}]$ enhancements in intrinsic AGB carbon stars (Jorissen et al. 1992; Abia et al. 2009, 2010, 2011, 2019) and metal-poor extrinsic stars (Lucatello et al. 2011; Abia et al. 2019).

Asymptotic giant branch stars consist of a degenerate $\mathrm{C}-\mathrm{O}$ core surrounded by a He-shell and a $\mathrm{H}$-shell that are separated by a He-rich intermediate zone (He-intershell), and an extended convective envelope. Recurrently, on a timescale of tens of thousands of years of quiescent $\mathrm{H}$-shell burning, the He-burning shell becomes thermally unstable. The large amount of energy released during this thermal pulse (TP), mainly due to the triple- $\alpha$ reaction, induces convective motions throughout the He-intershell and causes an expansion and thus a cooling of the
H-burning shell. As a consequence, the latter is switched off until the star contracts, and the rising temperature is high enough to reignite it. This process repeats until the complete erosion of the envelope by stellar winds. It characterizes the thermally pulsing AGB (TP-AGB) phase of these stars (e.g., Iben \& Renzini 1983). During the expansion of the envelope, convection may penetrate deep into the $\mathrm{H}-\mathrm{He}$ discontinuity beyond the H-burning shell and carry fresh products of the nucleosynthesis to the surface. This phenomenon is known as third dredge-up (TDU). At each TDU episode, protons are partially mixed from the envelope into the He-intershell, and as the $\mathrm{H}$-burning shell reignites, they are captured by the abundant ${ }^{12} \mathrm{C}$ nuclei to produce a ${ }^{13} \mathrm{C}$-enriched layer, the so-called ${ }^{13} \mathrm{C}$ pocket. ${ }^{13} \mathrm{C}$ nuclei are then efficiently burned through the ${ }^{13} \mathrm{C}(\alpha, \mathrm{n}){ }^{16} \mathrm{O}$ reaction at $T \approx 9 \times 10^{7} \mathrm{~K}$ (see Cristallo et al. 2018 and references therein) and so produce the free neutrons necessary for the synthesis of heavy elements in low-mass AGB stars $\left(M \lesssim 3 M_{\odot}\right)$ through the so-called $s$ (low)process (see Busso et al. 1999; Herwig 2005; Straniero et al. 2006; Karakas \& Lattanzio 2014 for reviews). A second neutron burst is driven by the ${ }^{22} \mathrm{Ne}(\alpha, \mathrm{n})^{25} \mathrm{Mg}$ reaction, which is efficiently activated at the base of the convective zone produced by a TP when the temperature reaches about $3 \times 10^{8} \mathrm{~K}$ (mostly in more massive AGBs; see, e.g., Karakas \& Lattanzio 2014).

During the interpulse phase, part of the neutrons released within the He-intershell are captured by ${ }^{14} \mathrm{~N}$ through the ${ }^{14} \mathrm{~N}(\mathrm{n}, \mathrm{p}){ }^{14} \mathrm{C}$ reaction to synthesize ${ }^{15} \mathrm{~N}$ by means of the chain ${ }^{14} \mathrm{C}(\alpha, \gamma){ }^{18} \mathrm{O}(\mathrm{p}, \alpha){ }^{15} \mathrm{~N} .{ }^{15} \mathrm{~N}$ is then burnt to primary ${ }^{19} \mathrm{~F}$ through the ${ }^{15} \mathrm{~N}(\alpha, \gamma){ }^{19} \mathrm{~F}$ reaction in the subsequent convective TP (Goriely \& Mowlavi 2000; Lugaro et al. 2004; Cristallo et al. 2014). An additional contribution to fluorine production comes from secondary ${ }^{13} \mathrm{C}$ and ${ }^{14} \mathrm{~N}$ synthesized in the H-burning ashes 
and from the eventual unburnt ${ }^{13} \mathrm{C}$ in the pocket (Cristallo et al. 2009a). In both cases, ${ }^{13} \mathrm{C}$ is engulfed in the convective shell generated by the TP and produces further secondary ${ }^{19} \mathrm{~F}$ through the same reaction chains. Fluorine is then carried to the surface by convective motions during the TDU. Therefore its envelope abundance is expected to be correlated with the abundances of carbon and $s$-process elements (see Abia et al. 2019 and references therein).

One of the most debated topics in AGB modeling is the formation of the ${ }^{13} \mathrm{C}$ pocket. The solution to this problem is strictly connected to a deep understanding of the physical processes governing mass-exchange at the interface of the convective envelope and the radiative core. Latest research has focused on studying nonconvective transport mechanisms that are usually ignored by the canonical theory of stellar structure and evolution. Different types of additional transport processes were invoked for the penetration of proton-rich material from the convective envelope into the He-intershell: convective overshoot (Herwig et al. 1997), opacity-induced overshoot (Cristallo et al. 2009a, 2011, 2015b), mixing induced by rotation (Herwig et al. 2003; Siess et al. 2004; Piersanti et al. 2013) or internal gravity waves (Denissenkov \& Tout 2003; Battino et al. 2016), and mixing induced by magnetic buoyancy (Trippella et al. 2014, 2016). Focusing on the production of fluorine, it was suggested that a mechanism leading to the formation of an extended ${ }^{13} \mathrm{C}$ pocket and at the same time, to a small amount of ${ }^{14} \mathrm{~N}$, could solve the problem of ${ }^{19} \mathrm{~F}$ overproduction with respect to $s$-elements in lowmass metal-poor objects (Abia et al. 2019).

Post-process neutron-capture models for AGB stars in which the formation of the required ${ }^{13} \mathrm{C}$ reservoir is ascribed to mixing induced by magnetic buoyancy were shown to be able to account for the solar distributions of $s$-only isotopes (Trippella et al. 2016), isotopic ratios of $s$-elements measured in presolar SiC grains (Palmerini et al. 2018), and for a large part of the abundance observations in evolved low-mass stars (Busso et al. 2021). The theory of mixing triggered by magnetic buoyancy developed by Nucci \& Busso (2014) was used for this. The latter has recently been adopted by Vescovi et al. (2020), who contextualized magnetic mixing in the more complex framework of FRUITY stellar evolutionary models, obtaining a satisfactory fit to presolar grain isotopic ratios. The same stellar models also provide a consistent explanation for the observed yttrium abundance trends in the inner region of the Galactic disk (Magrini et al. 2021). FRUITY magnetic models predict that deep profiles of low proton abundances are generated below the convective envelope border. The low proton concentration severely limits local ${ }^{14} \mathrm{~N}$ formation because protons are almost entirely consumed for the synthesis of ${ }^{13} \mathrm{C}$, thereby affecting ${ }^{19} \mathrm{~F}$ production as well.

We investigate fluorine nucleosynthesis in low-mass AGB stars by computing a new series of stellar models accounting for the formation of a ${ }^{13} \mathrm{C}$ pocket induced by magnetic buoyancy. The structure of the paper is as follows. In Sect. 2 we present the stellar evolutionary code, the nuclear network, and the mixing algorithm adopted to calculate the AGB models. In Sect. 3 we compare our models with available $F$ spectroscopic abundances in a sample of Galactic and extragalactic AGB carbon stars, as well as with other metal-poor Ba-type and CH-type evolved stars. Finally, we summarize our conclusions in the last section.

\section{Stellar models}

The stellar models presented in this work were computed using the FUNS code (see Straniero et al. 2006 and references therein)
Table 1. Reaction rates of relevance to fluorine nucleosynthesis.

\begin{tabular}{lc}
\hline \hline Reaction & Reference \\
\hline${ }^{14} \mathrm{~N}(\mathrm{p}, \gamma){ }^{15} \mathrm{O}$ & 1 \\
${ }^{15} \mathrm{~N}(\mathrm{p}, \gamma){ }^{16} \mathrm{O}$ & 2 \\
${ }^{17} \mathrm{O}(\mathrm{p}, \gamma){ }^{18} \mathrm{~F}$ & 3 \\
$\left.{ }^{18} \mathrm{O}(\mathrm{p}, \gamma)\right)^{19} \mathrm{O}$ & 4 \\
$\left.{ }^{15} \mathrm{~N}(\mathrm{p}, \alpha)\right)^{12} \mathrm{C}$ & 5 \\
${ }^{17} \mathrm{O}(\mathrm{p}, \alpha){ }^{14} \mathrm{~N}$ & 6 \\
${ }^{18} \mathrm{O}(\mathrm{p}, \alpha){ }^{15} \mathrm{~N}$ & 7 \\
${ }^{19} \mathrm{~F}(\mathrm{p}, \alpha){ }^{15} \mathrm{O}$ & 8 \\
${ }^{14} \mathrm{C}(\alpha, \gamma){ }^{18} \mathrm{O}$ & 9 \\
${ }^{14} \mathrm{~N}(\alpha, \gamma){ }^{18} \mathrm{~F}$ & 10 \\
${ }^{15} \mathrm{~N}(\alpha, \gamma){ }^{19} \mathrm{~F}$ & 10 \\
${ }^{18} \mathrm{O}(\alpha, \gamma){ }^{22} \mathrm{Ne}$ & 10 \\
${ }^{19} \mathrm{~F}(\alpha, \mathrm{p}){ }^{22} \mathrm{Ne}$ & 11 \\
${ }^{13} \mathrm{C}(\alpha, \mathrm{n}){ }^{16} \mathrm{O}$ & 12 \\
${ }^{14} \mathrm{~N}(\mathrm{n}, \mathrm{p})^{14} \mathrm{C}$ & 13 \\
\hline
\end{tabular}

References. (1) Imbriani et al. (2005); (2) Leblanc et al. (2010); (3) Di Leva et al. (2014); (4) Best et al. (2019); (5) Angulo et al. (1999); (6) Bruno et al. (2016); (7) Bruno et al. (2019); (8) Indelicato et al. (2017); (9) Johnson et al. (2009); (10) Iliadis et al. (2010); (11) D'Agata et al. (2018); (12) Trippella \& La Cognata (2017); (13) Wallner et al. (2016).

Table 2. Initial mass, $[\mathrm{Fe} / \mathrm{H}]$, total metallicity (including $\alpha_{\mathrm{O}-\mathrm{Ca}}$ enhancements), and helium.

\begin{tabular}{lccccc}
\hline \hline Mass $\left(M_{\odot}\right)$ & {$[\mathrm{Fe} / \mathrm{H}]$} & $Z$ & $Y$ & $\alpha_{\mathrm{O}}$ & $\alpha_{\mathrm{Ne}-\mathrm{Ca}}$ \\
\hline 1.5 & -2.18 & $3.10 \times 10^{-4}$ & 0.247 & 0.7 & 0.4 \\
1.5 & -1.88 & $5.33 \times 10^{-4}$ & 0.248 & 0.6 & 0.4 \\
1.5 & -1.70 & $8.00 \times 10^{-4}$ & 0.248 & 0.6 & 0.4 \\
1.5 & -1.18 & $2.13 \times 10^{-3}$ & 0.250 & 0.5 & 0.4 \\
2.0 & -0.70 & $5.70 \times 10^{-3}$ & 0.254 & 0.4 & 0.3 \\
2.0 & -0.40 & $8.13 \times 10^{-3}$ & 0.257 & 0.2 & 0.15 \\
2.0 & -0.18 & $1.00 \times 10^{-2}$ & 0.259 & 0 & 0 \\
2.0 & +0.05 & $1.67 \times 10^{-2}$ & 0.267 & 0 & 0 \\
2.0 & +0.13 & $2.00 \times 10^{-2}$ & 0.271 & 0 & 0 \\
\hline
\end{tabular}

by following the chemical evolution of approximately 500 isotopes (from hydrogen to bismuth) linked by more than 800 reactions (charged-particle reactions, neutron captures, and $\beta$ decays). The baseline nuclear network is essentially the same as was described in Cristallo et al. (2009a), with the addition of some recently updated reaction rates. In particular, the list of adopted reaction rates relevant to the nucleosynthesis of fluorine is reported in Table 1.

For radiative opacities, we computed opacity tables by means of the OPAL Web tool ${ }^{1}$. At the low temperatures $(\log T \leq 4.05)$ that are typical of the external layers of stars, we used the ÆSOPUS tool (Marigo \& Aringer 2009), which includes molecular and atomic species relevant for AGB atmospheres to compute the opacity tables. In particular, ÆSOPUS allows taking changes in opacity into account that are due to eventual oxygen and $\alpha$-enhancement (see Table 2) and to chemical composition variations in the envelope when the star becomes carbon rich (Marigo 2002; Cristallo et al. 2007; Ventura \& Marigo 2010).

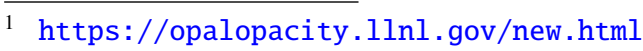


The opacity tables used in this work were calculated with the scaled-solar element distribution by Lodders (2020). Accordingly, a solar-calibrated value of the mixing length parameter $\alpha_{\mathrm{ml}}=1.86$ was adopted (see Vescovi et al. 2019 for more details). Regarding the mass-loss rate, we adopted a Reimers formula with $\eta=0.4$ for the pre-AGB evolution and the rate used by Abia et al. (2020) for the AGB phase (this rate is slightly different from that of Straniero et al. 2006 because they adopted recalculated bolometric corrections).

The surface enrichment of $\mathrm{C}$ and $s$-process elements in AGB stars is related to the complex coupling between convective mixing and nuclear processes. The problem of the neutron source in AGB stars, and in particular, the physical process driving the formation of a ${ }^{13} \mathrm{C}$ pocket in the He-rich intershell, is still a matter of debate (see Sect. 1). In previous FRUITY models, the partial mixing of hydrogen from the envelope that is required to produce fresh ${ }^{13} \mathrm{C}$ was accounted for by the so-called opacity-induced overshoot (Straniero et al. 2006; Cristallo et al. 2009a). This powers an additional mixing and the formation of a chemically smooth transition zone between the fully convective envelope and the radiative region. At the inner border of the convective envelope, the velocity of the descending material accelerated by convection is estimated as

$v=v_{\mathrm{cb}} \exp \left(-\frac{\delta r}{\beta H_{\mathrm{P}}}\right)$,

where $v_{\mathrm{cb}}$ is the velocity at the convective border, $\delta r$ is the distance from the border, $H_{\mathrm{P}}$ is the pressure scale height at the convective border, and $\beta$ is a free parameter whose value was set to 0.1 in order to maximize the $s$-process production in low-mass AGB stars (see Cristallo et al. 2009a; Guandalini \& Cristallo 2013). The free parameter $\beta$ regulates the number of protons that is mixed beyond the bare convective border, and it also affects the TDU efficiency. In standard FRUITY models, the partially mixed zone is extended below the formal Schwarzschild convective boundary down to $2 H_{\mathrm{P}}$ (Straniero et al. 2006). Instead, in FRUITY Tail models (Cristallo et al. 2015a), the penetration limit is fixed to the layer where the convective velocity is $10^{-11}$ times lower than the value attained at the Schwarzschild border. The ensuing ${ }^{13} \mathrm{C}$ pockets were shown to be remarkably larger than those obtained in standard FRUITY models, which results in a substantial increase in surface $s$-process enrichment.

Vescovi et al. (2020) found that when updated physical inputs are included, the slope of the exponential decline of convective velocities has to be reduced to $\beta=0.025$ to avoid altering the efficiency of the TDU and obtain a sizable amount of dredged-up material. However, models computed with this parameter choice show additional mixing that is so inefficient to almost inhibit the production of $s$-process nuclei. Thus, mixing triggered by magnetic fields has been introduced as an additional mixing mechanism. Vescovi et al. (2020) assumed that a toroidal field is present in the radiative He-intershell at the beginning of the TDU and triggers the buoyant rise of magnetic flux tubes. As a consequence, a matter flow is pushed to the envelope. For mass conservation, this induces a matter downflow of H-rich material to the He-intershell that is required for the formation of the ${ }^{13} \mathrm{C}$ pocket. The downflow velocity can be expressed as

$v(r)=u_{\mathrm{p}}\left(\frac{r_{\mathrm{p}}}{r}\right)^{k+2}$,

where $r_{\mathrm{p}}$ is the distance from the stellar center from which magnetic flux tubes, generated in the He-intershell, start to rise. $r_{\mathrm{p}}$ can be identified from the critical toroidal $B_{\varphi}$ necessary for the onset of magnetic buoyancy instabilities (Vescovi et al. 2020). $k$ is the exponent quantifying the density decline, being $\rho(r) \propto r^{k}$, in the He-rich radiative layers below the convective envelope during a TDU, and it is typically lower than -3 (see also Busso et al. 2021). The identification of the critical field necessary for the occurrence of instabilities by magnetic buoyancy allows identifying the corresponding radial position $r_{\mathrm{p}}$ from which magnetic structures arise. $u_{\mathrm{p}}$ is the effective starting buoyant velocity. The magnetized domains are rather fast and occupy only a small fraction of the total mass of the stellar layer $\left(\simeq 1 / 10^{5}\right.$; see, e.g., Busso et al. 2007; Trippella et al. 2016), thus transporting mass at a rate equal to assuming an effective buoyant velocity for all the material in the layer. Vescovi et al. (2020) found that most of the heavy-element isotope ratios measured in presolar $\mathrm{SiC}$ grains from AGB stars are consistently explained by stellar AGB models computed with a unique choice for the toroidal field strength and the initial buoyant velocity, namely $B_{\varphi}=5 \times 10^{4} \mathrm{G}$ and $u_{\mathrm{p}}=5 \times 10^{-5} \mathrm{~cm} \mathrm{~s}^{-1}$. Nonetheless, we expect a nontrivial mass-metallicity dependence of the mixing triggered by magnetic buoyancy (as for other type of mixing; see, e.g., Joyce \& Chaboyer 2018; Battino et al. 2021). In particular, the toroidal field strength is determined by the differential rotation profile established in the He-intershell that amplifies the magnetic field through dynamo action (see Sect. 4). In this framework, the compactness of a star at the pre-AGB and AGB stages is a fundamental property that affects the dynamo process and the following mixing induced by magnetic buoyancy. The stellar models presented here have $\mathrm{H}$-exhausted core masses at the beginning of the TP-AGB phase very similar to the $2 M_{\odot}$ closeto-solar metallicity models presented in Vescovi et al. (2020). We therefore adopted for all the computed models their parameter choice for $B_{\varphi}$ and $u_{\mathrm{p}}$ without further adjustments. We defer the analysis of the dependence of the magnetic mixing on the stellar core mass to a future work.

\section{Results and discussion}

In the following, we compare literature data and new FRUITY magnetic models. To do this, we adopted data from previous studies of intrinsic AGB carbon stars (Abia et al. 2010, 2011, 2015, 2019) and extrinsic CH/Ba stars (Lucatello et al. 2011). In Fig. 1 we report the $[\mathrm{F} / \mathrm{Fe}]$ ratios of our selected sample as a function of the iron content $[\mathrm{Fe} / \mathrm{H}]$. We plot four group data: galactic (N-type) carbon stars, SC-type stars, extragalactic carbon stars, and extrinsic $\mathrm{CH} / \mathrm{Ba}$ stars. Observational data are compared with stellar models of $1.5 M_{\odot}$ and $2 M_{\odot}$ at different TPs. Within the observational errors there is a good agreement, confirming the expected F-enhancement trend with the metallicity (see e.g. Lugaro et al. 2004; Cristallo et al. 2014). One word of caution has to be used regarding fluorine abundances in extrinsic $\mathrm{CH} / \mathrm{Ba}$ stars, for which $[\mathrm{F} / \mathrm{Fe}]$ ratios only represent lower limits due to dilution effects of binary mass-transfer phenomena (Abia et al. 2019).

The top panel of Fig. 2 shows the comparison between theoretical predictions of FRUITY magnetic models with initial mass $M=2 M_{\odot}$ and $[\mathrm{Fe} / \mathrm{H}] \geq-0.4$ and spectroscopic observations for $[\mathrm{F} / \mathrm{Fe}]$ ratios versus the average $s$-element enhancement. Four observational indices are commonly used to represent $s$ process distributions and overabundances: [1s/Fe] (representative of the first $s$-process peak), [hs/Fe] (representative of the second $s$-process peak), [s/Fe], and [hs/ls]. Given the inhomogeneity of the available elemental abundance of heavy $s$-elements for the selected sample, we used the mean of the relative abundances of only $\mathrm{Y}$ and $\mathrm{Zr}$ to express the 1s-index, and $\mathrm{Ba}$ and $\mathrm{La}$ to 


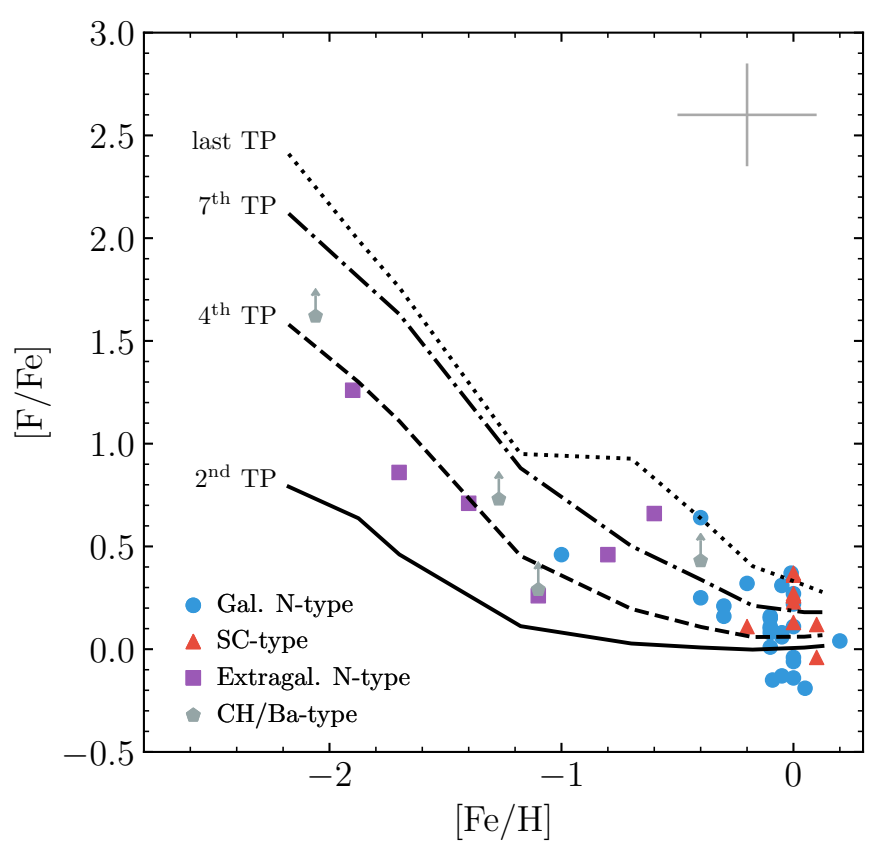

Fig. 1. Comparison of observed $[\mathrm{F} / \mathrm{Fe}]$ ratios as a function of the metallicity and FRUITY magnetic models. Symbols refer to the four data groups: circles represent galactic carbon stars, triangles show SC-type stars, squares stand for extragalactic carbon stars, and pentagons show extrinsic $\mathrm{CH} / \mathrm{Ba}$ stars. The lines represent theoretical predictions for 2 (for $[\mathrm{Fe} / \mathrm{H}] \geq-0.7)$ and $1.5 M_{\odot}($ for $[\mathrm{Fe} / \mathrm{H}]<-0.7)$ AGB stars at different TPs. A typical error bar is indicated.

express the hs-index. The difference between the hs- and the ls-index quantifies the $s$-element index [hs/ls], while the total average $s$-element enhancement is given by the mean of the relative abundances of $\mathrm{Y}, \mathrm{Zr}, \mathrm{Ba}$, and $\mathrm{La}$. For close-to-solar metallicity galactic N-type carbon stars (dots) and SC-type stars (triangles), the observed increase in fluorine with $s$-element enhancement is well reproduced by theoretical models. The predicted $[\mathrm{F} / \mathrm{Fe}]$ are always positive because ${ }^{19} \mathrm{~F}$ is overall produced in low-mass AGB stars (see also Lugaro et al. 2004). On the other hand, a few observational data show negative values that are still consistent within the errors with no fluorine production, however. The comparison with N-type and extrinsic $\mathrm{CH} / \mathrm{Ba}$ type stars (pentagon) with $[\mathrm{Fe} / \mathrm{H}]<-0.2$ is more challenging because less information is available at these metallicities. In particular, two out of the three stars appear to exhibit the same trend as is observed at higher metallicity and agree well with theoretical predictions, while the fluorine and $s$-elements abundances derived for TX Psc are slightly out of the trend and are not fully reproduced by stellar models. This interpretation is confirmed even when $[\mathrm{F} / \mathrm{s}]$ ratios are considered (see the bottom panel of Fig. 2). This index shows that the fluorine enhancement for the extrinsic stars is not affected by the uncertainties related to the dilution factor and provides a more robust tool for comparison. Confirming the previous analysis, models are able to replicate the quasi-linear decreasing trend of $[\mathrm{F} / \mathrm{s}]$ with the surface $s$-process enrichment, except for the marginally outside TX Psc. Given the typical uncertainties affecting observational data, however, it is difficult to firmly conclude that model predictions and observations disagree for this single star.

In our computations we used the ${ }^{13} \mathrm{C}(\alpha, \mathrm{n}){ }^{16} \mathrm{O}$ reaction rate provided by Trippella \& La Cognata (2017), which combined the latest asymptotic normalization coefficient values and the

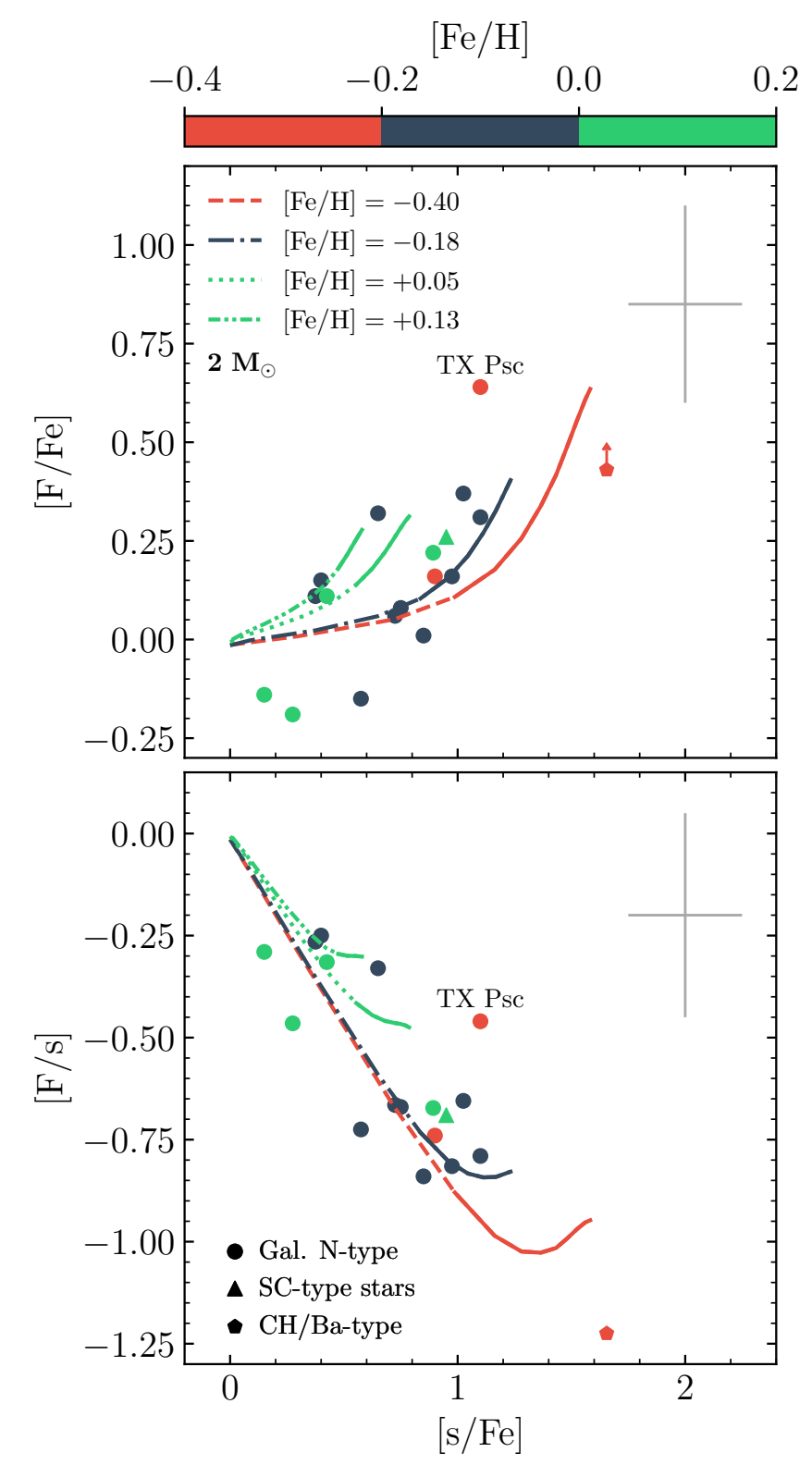

Fig. 2. Observed $[\mathrm{F} / \mathrm{Fe}]$ (top panel) and $[\mathrm{F} / \mathrm{s}]$ (bottom panel) vs. average $s$-element enhancements compared with theoretical predictions for $2 M_{\odot}$ and close-to-solar metallicity models. Symbols are the same as in Fig. 1. Data points and theoretical lines are color-coded by $[\mathrm{Fe} / \mathrm{H}]$. The continuous portion of the lines represents the theoretical C-rich phase, and the discontinuous portion represents the O-rich phase. Typical uncertainties are shown. See text for details.

Trojan horse method, to determine the astrophysical $S$-factor with a indirect approach. At the typical temperature of $\sim 90 \mathrm{MK}$ corresponding to the radiative ${ }^{13} \mathrm{C}$ burning phase, the most recent direct measurement, given by Heil et al. (2008), is almost $20 \%$ higher. Adopting this rate has no effect on the production of $s$-nuclei if the ${ }^{13} \mathrm{C}$ pocket is fully consumed during the interpulse period (see also Trippella \& La Cognata 2017), however. Instead, if some amount of ${ }^{13} \mathrm{C}$ survives due to the lower reaction rate, it will be swallowed by the convective shell generated by the following TP and burnt at a rather high temperature ( 200 MK). This provides an additional neutron burst and possibly affects the production of $s$-nuclei and ${ }^{19} \mathrm{~F}$. This burst was shown to occur only during the very first TPs in low-mass AGB stars with high metallicity $(Z \geq 0.01$; see Cristallo et al. 
2009a; Karakas 2010). In our $2 M_{\odot} Z=0.02$ model, switching from the Trippella \& La Cognata (2017) to the Heil et al. (2008) reaction rate produces variations of less than $3 \%$ in the $[\mathrm{F} / \mathrm{Fe}]$, $[\mathrm{ls} / \mathrm{Fe}]$, and $[\mathrm{hs} / \mathrm{Fe}]$ indexes, which is much lower than the typical uncertainties affecting spectroscopic observations of $s$-processrich stars. Stellar models of lower metallicity are expected to produce even minor variations (Cristallo et al. 2009a; Karakas 2010) unless their mass and metallicity are so low $(M \lesssim$ $1.3 M_{\odot}$ and $\left.[\mathrm{Fe} / \mathrm{H}] \lesssim-2.5\right)$ that they experience a proton ingestion episode at the first fully developed TP (see Cristallo et al. 2009b; Campbell et al. 2010; Choplin et al. 2021).

In Figs. 3 and 4 we perform a similar comparison at low metallicities. Because there is no homogeneous sample of stars with both $\mathrm{Ba}$ and $\mathrm{La}$, we compared the model predictions separately. For both figures, we present in different panels theoretical expectations from new "FRUITY Magnetic" models (top panels), from standard "FRUITY" models (middle panels) and from "FRUITY Tail" models (bottom panels). Magnetic models are capable of reproducing the observed spread well at different metallicities for the $[\mathrm{F} / \mathrm{Ba}]$ and $[\mathrm{F} / \mathrm{La}]$ ratios as a function of the corresponding overall $s$-process enhancement. Conversely, standard FRUITY models fail in reproducing both the $\mathrm{Ba}$ and La enrichment, as well as the $[\mathrm{F} / \mathrm{Ba}]$ and $[\mathrm{F} / \mathrm{La}]$ ratios. Finally, FRUITY Tail models are sufficiently enriched in $s$-elements because the ${ }^{13} \mathrm{C}$ pockets are larger than those obtained in standard FRUITY models, but they still show a systematic overproduction of fluorine with respect to $\mathrm{Ba}$ and La. As a whole, FRUITY magnetic models show a reduction of fluorine production that agrees well with spectroscopic observations for lowmetallicity stars.

As previously mentioned, ${ }^{19} \mathrm{~F}$ is primarily synthesized in AGB stars through the ${ }^{15} \mathrm{~N}(\alpha, \gamma){ }^{19} \mathrm{~F}$ reaction reaction in the convective zone generated by a TP. The production of ${ }^{15} \mathrm{~N}$ in the He-intershell involves a complex nuclear chain of successive $\mathrm{n}$ - (or $\mathrm{p}$-) and $\alpha$-captures starting from ${ }^{13} \mathrm{C}$ nuclei. A first source of ${ }^{13} \mathrm{C}$ is represented by the ${ }^{13} \mathrm{C}$ pocket itself, whose radiative burning leads to the accumulation of ${ }^{15} \mathrm{~N}$. A second source is the ${ }^{13} \mathrm{C}$ left in the H-burning shell ashes, which is engulfed in the convective zone generated by the TP and is rapidly burned through the ${ }^{13} \mathrm{C}(\alpha, \mathrm{n}){ }^{16} \mathrm{O}$ reaction. In standard FRUITY models, the two channels contribute almost equally to the fluorine nucleosynthesis, especially at close-to-solar metallicity. On the other hand, at low metallicities, the contribution of ${ }^{13} \mathrm{C}$ left in the H-burning ashes is lower (Cristallo et al. 2009a). Instead, in FRUITY Tail models, the larger ${ }^{13} \mathrm{C}$ pockets guarantee a net increase of $s$-process abundances and a large fluorine decrease at fixed $s$-process surface enhancement (Abia et al. 2015). However, these models barely reach negative values for $[\mathrm{F} / \mathrm{Ba}]$ and [F/La] (see the lower panels of Figs. 3 and 4), which indicates that fluorine production needs to be further suppressed without altering the $s$-process enrichment (see also Abia et al. 2019). The extended profile and the low proton abundance characterizing FRUITY magnetic models (Vescovi et al. 2020) have the twofold effect of forming large ${ }^{13} \mathrm{C}$ pockets and reducing the formation of primary ${ }^{14} \mathrm{~N}$. In this framework, the few available protons first produce ${ }^{13} \mathrm{C}$ through the ${ }^{12} \mathrm{C}(\mathrm{p}, \gamma){ }^{13} \mathrm{C}$ reaction, preventing more proton captures from forming ${ }^{14} \mathrm{~N}$ and thereby inhibiting the nuclear chain ${ }^{14} \mathrm{~N}(\mathrm{n}, \mathrm{p}){ }^{14} \mathrm{C}(\alpha, \gamma){ }^{18} \mathrm{O}(\mathrm{p}$, $\alpha)^{15} \mathrm{~N}(\alpha, \gamma){ }^{19} \mathrm{~F}$. Hence, any fluorine appearing in AGB envelopes in these models is of secondary nature, generated by ${ }^{14} \mathrm{~N}$ concentrations left behind by $\mathrm{H}$-shell burning. This results in low fluorine enhancements and high $s$-enhancements, which places FRUITY magnetic models in close agreement with observations in very metal-poor AGB stars.

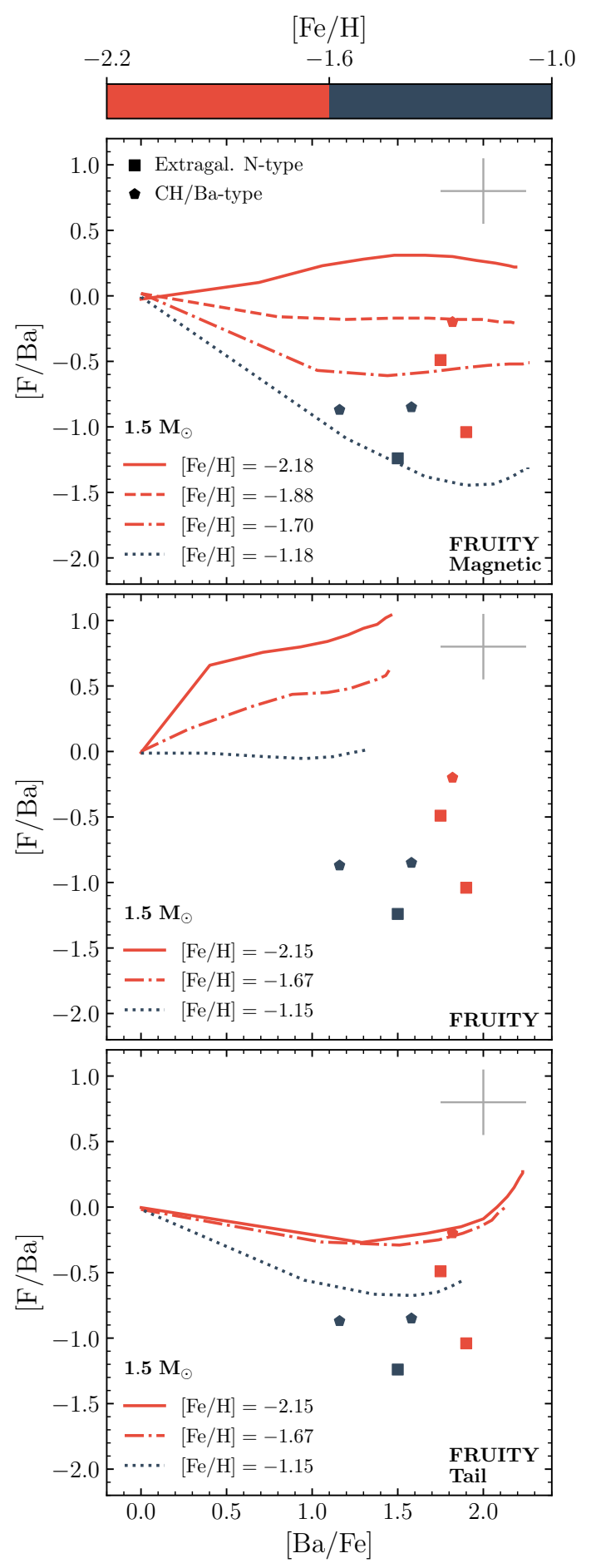

Fig. 3. $[\mathrm{F} / \mathrm{Ba}]$ vs. $[\mathrm{Ba} / \mathrm{Fe}]$ in the sample stars with $[\mathrm{Fe} / \mathrm{H}] \leq-1.0$. The squares show extragalactic carbon stars, and the pentagons represent extrinsic $\mathrm{CH} / \mathrm{Ba}$ stars. The lines are theoretical predictions for $1.5 M_{\odot}$ TP-AGB stars with low metallicity, assuming different mechanisms for the formation of the ${ }^{13} \mathrm{C}$ pocket (see text for details). At these low metallicities, theoretical AGB models predict that the star becomes C-rich from first TDU episodes. Data points and theoretical lines are colorcoded by $[\mathrm{Fe} / \mathrm{H}]$. Typical error bars are indicated.

\section{Conclusions}

The fluorine production in low-mass AGB stars has been revisited in the light of upgraded FRUITY stellar models in which the ${ }^{13} \mathrm{C}$ neutron source is related to phenomena induced by magnetic 


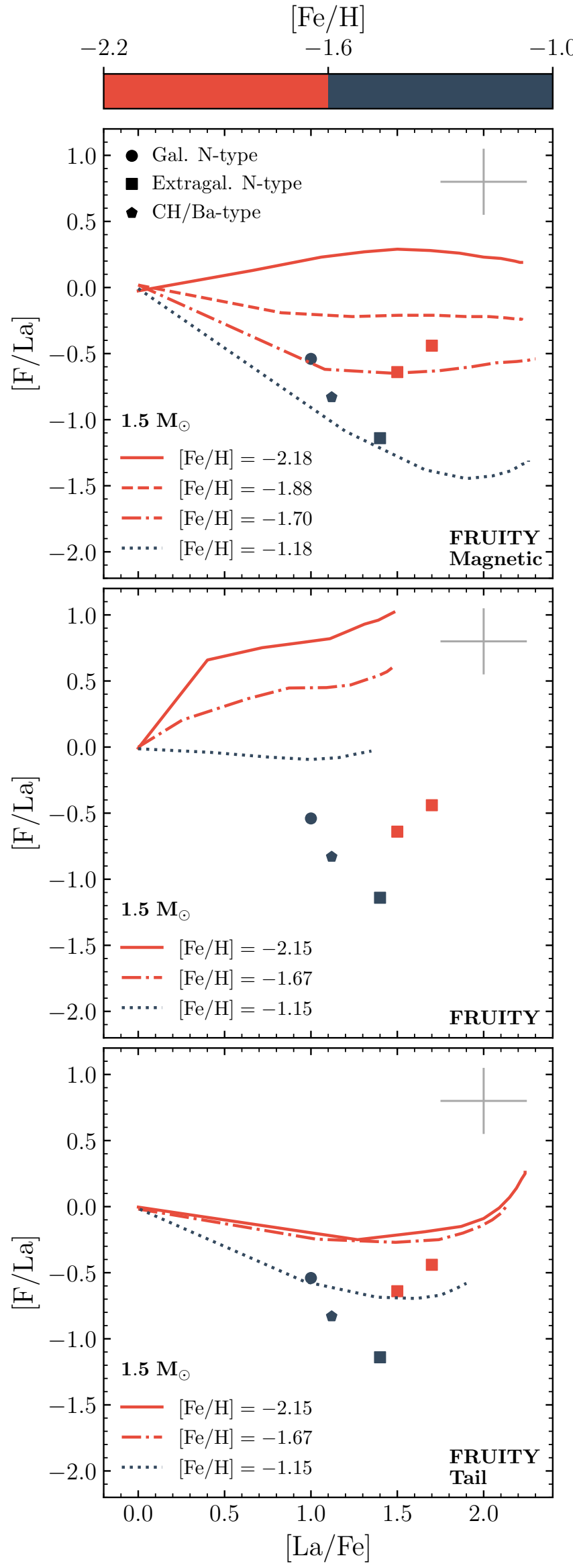

Fig. 4. Same as Fig. 3, but for $[\mathrm{F} / \mathrm{La}]$ vs. $[\mathrm{La} / \mathrm{Fe}]$. buoyancy. The predicted fluorine enhancements agree with those observed in carbon stars at different metallicities. The observed correlation between $\mathrm{F}$ and the $s$-element enhancements is also reproduced. On one hand, new FRUITY magnetic models show a reduced net ${ }^{19} \mathrm{~F}$ production with respect to models in which the partial mixing of hydrogen during a TDU is attributed to overshooting below the convective envelope of an AGB star. This is a consequence of the low abundance of ${ }^{14} \mathrm{~N}$ in the ${ }^{13} \mathrm{C}$ pocket, which leads to a negligible production of fluorine during the ${ }^{13} \mathrm{C}$ radiative burning. The ${ }^{19} \mathrm{~F}$ envelope abundance is therefore only ascribed to the amount of the secondary ${ }^{13} \mathrm{C}$ in the $\mathrm{H}$-shell ashes, which depends on the $\mathrm{CNO}$ abundances in the star. On the other hand, mixing induced by magnetic buoyancy leads to extended ${ }^{13} \mathrm{C}$ pockets, which results in high surface $s$-process enrichments. As a whole, the new FRUITY magnetic models simultaneously account for the observed fluorine and the average $s$-element enhancements in intrinsic AGB carbon stars and extrinsic $\mathrm{CH} / \mathrm{Ba}$ stars.

Different types of data (presolar grain measurements and spectroscopic observations) related to stars belonging to different components of our Galaxy (disk and halo) indicate a single configuration of mixing induced by magnetic buoyancy. This scenario suggests that magnetism probably also is a quite common phenomenon in evolved low-mass stars. In this respect, several observations of magnetic fields of a few $G$ in the envelopes of AGB stars have been made (see, e.g., Vlemmings 2019 and references therein). Another piece of evidence for magnetic fields in low-mass stars is provided by the increasing number of observed magnetic white dwarfs (WDs). During the least decades, many WDs lying within 20 pc from the Sun were shown to have a field strength above $\sim 1 \mathrm{MG}$, while the available data were not sufficient to asses the presence or absence of a magnetic field for most of them. These studies suggested that about $10 \%$ of all WDs are magnetic (e.g., Kawka et al. 2007). However, the advent of increasingly precise spectroscopic and spectropolarimetric observations, which improve the capability of detecting magnetic fields of low strength, revealed that at least $\sim 20 \%$ of the WDs possess magnetic fields ranging from a few $\mathrm{kG}$ to about 1000 MG (Landstreet \& Bagnulo 2019; Bagnulo \& Landstreet 2020), indicating that magnetism is a widespread and not a rare property of WDs. These magnetic fields are most likely the remain of previous phases, and they may be the result of a complicated interaction between fossil and dynamo generated fields during stellar evolution and/or stellar merger events (Ferrario et al. 2020). In our scenario, we propose that below the extended convective envelope of low-mass AGB stars, strong toroidal magnetic fields exist and trigger a mixing induced by magnetic buoyancy that is adequate for the formation of the ${ }^{13} \mathrm{C}$ pocket. The required mixing rate places constraints on the field strength. In this picture, the toroidal field is assumed to originate from a dynamo operating in the AGB interiors that amplifies a small seed poloidal field by draining the available differential rotation energy.

This study provides another independent confirmation (see Magrini et al. 2021) that the calibration performed by Vescovi et al. (2020) on the free parameters characterizing mixing induced by magnetic buoyancy is extremely robust. However, additional analyses are needed to validate this statement before we can claim a definitive theory.

Acknowledgements. DV and SC acknowledge S. Bagnulo for fruitful discussions. DV acknowledges financial support from the German-Israeli Foundation (GIF No. I-1500-303.7/2019). CA acknowledges financial support from the 
Agencia Estatal de Investigación of the Spanish Ministerio de Ciencia e Innovación through the FEDER founds projects PGC2018-095317-B-C2.

\section{References}

Abia, C., Recio-Blanco, A., de Laverny, P., et al. 2009, ApJ, 694, 971

Abia, C., Cunha, K., Cristallo, S., et al. 2010, ApJ, 715, L94

Abia, C., Cunha, K., Cristallo, S., et al. 2011, ApJ, 737, L8

Abia, C., Cunha, K., Cristallo, S., \& de Laverny, P. 2015, A\&A, 581, A88

Abia, C., Cristallo, S., Cunha, K., de Laverny, P., \& Smith, V. V. 2019, A\&A, 625, A40

Abia, C., de Laverny, P., Cristallo, S., Kordopatis, G., \& Straniero, O. 2020 A\&A, 633, A135

Angulo, C., Arnould, M., Rayet, M., et al. 1999, Nucl. Phys. A, 656, 3

Bagnulo, S., \& Landstreet, J. D. 2020, A\&A, 643, A134

Battino, U., Pignatari, M., Ritter, C., et al. 2016, ApJ, 827, 30

Battino, U., Lederer-Woods, C., Cseh, B., Denissenkov, P., \& Herwig, F. 2021, Universe, 7, 25

Best, A., Pantaleo, F. R., Boeltzig, A., et al. 2019, Phys. Lett. B, 797, 134900

Bruno, C. G., Scott, D. A., Aliotta, M., et al. 2016, Phys. Rev. Lett., 117, 142502

Bruno, C. G., Aliotta, M., Descouvemont, P., et al. 2019, Phys. Lett. B, 790, 237

Busso, M., Gallino, R., \& Wasserburg, G. J. 1999, ARA\&A, 37, 239

Busso, M., Wasserburg, G. J., Nollett, K. M., \& Calandra, A. 2007, ApJ, 671, 802

Busso, M., Vescovi, D., Palmerini, S., Cristallo, S., \& Antonuccio-Delogu, V. 2021, ApJ, 908, 55

Campbell, S. W., Lugaro, M., \& Karakas, A. I. 2010, A\&A, 522, L6

Choplin, A., Siess, L., \& Goriely, S. 2021, A\&A, 648, A119

Cristallo, S., Straniero, O., Lederer, M. T., \& Aringer, B. 2007, ApJ, 667, 489

Cristallo, S., Straniero, O., Gallino, R., et al. 2009a, ApJ, 696, 797

Cristallo, S., Piersanti, L., Straniero, O., et al. 2009b, PASA, 26, 139

Cristallo, S., Piersanti, L., Straniero, O., et al. 2011, ApJS, 197, 17

Cristallo, S., Di Leva, A., Imbriani, G., et al. 2014, A\&A, 570, A46

Cristallo, S., Abia, C., Straniero, O., \& Piersanti, L. 2015a, ApJ, 801, 53

Cristallo, S., Straniero, O., Piersanti, L., \& Gobrecht, D. 2015b, ApJS, 219, 40

Cristallo, S., La Cognata, M., Massimi, C., et al. 2018, ApJ, 859, 105

D’Agata, G., Pizzone, R. G., La Cognata, M., et al. 2018, ApJ, 860, 61

Denissenkov, P. A., \& Tout, C. A. 2003, MNRAS, 340, 722

Di Leva, A., Scott, D. A., Caciolli, A., et al. 2014, Phys. Rev. C, 89, 015803

Ferrario, L., Wickramasinghe, D., \& Kawka, A. 2020, Adv. Space Res., 66, 1025

Forestini, M., Goriely, S., Jorissen, A., \& Arnould, M. 1992, A\&A, 261, 157

Goriely, S., \& Mowlavi, N. 2000, A\&A, 362, 599

Grisoni, V., Romano, D., Spitoni, E., et al. 2020, MNRAS, 498, 1252

Guandalini, R., \& Cristallo, S. 2013, A\&A, 555, A120

Heil, M., Detwiler, R., Azuma, R. E., et al. 2008, Phys. Rev. C, 78, 025803

Herwig, F. 2005, ARA\&A, 43, 435

Herwig, F., Langer, N., \& Lugaro, M. 2003, ApJ, 593, 1056

Herwig, F., Bloecker, T., Schoenberner, D., \& El Eid, M. 1997, A\&A, 324, L81
Iben, I., Jr., \& Renzini, A. 1983, ARA\&A, 21, 271

Iliadis, C., Longland, R., Champagne, A. E., Coc, A., \& Fitzgerald, R. 2010, Nucl. Phys. A, 841, 31

Imbriani, G., Costantini, H., Formicola, A., et al. 2005, Eur. Phys. J. A, 25, 455 Indelicato, I., La Cognata, M., Spitaleri, C., et al. 2017, ApJ, 845, 19

Johnson, E. D., Rogachev, G. V., Mitchell, J., Miller, L., \& Kemper, K. W. 2009, Phys. Rev. C, 80, 045805

Jorissen, A., Smith, V. V., \& Lambert, D. L. 1992, A\&A, 261, 164

José, J., \& Hernanz, M. 1998, ApJ, 494, 680

Joyce, M., \& Chaboyer, B. 2018, ApJ, 856, 10

Karakas, A. I. 2010, MNRAS, 403, 1413

Karakas, A. I., \& Lattanzio, J. C. 2014, PASA, 31, e030

Kawka, A., Vennes, S., Schmidt, G. D., Wickramasinghe, D. T., \& Koch, R. 2007, ApJ, 654, 499

Kobayashi, C., Izutani, N., Karakas, A. I., et al. 2011, ApJ, 739, L57

Landstreet, J. D., \& Bagnulo, S. 2019, A\&A, 628, A1

Leblanc, P. J., Imbriani, G., Görres, J., et al. 2010, Phys. Rev. C, 82, 055804

Limongi, M., \& Chieffi, A. 2018, ApJS, 237, 13

Lodders, K. 2020, Solar Elemental Abundances (Oxford: Oxford University Press)

Lucatello, S., Masseron, T., Johnson, J. A., Pignatari, M., \& Herwig, F. 2011, ApJ, 729, 40

Lugaro, M., Ugalde, C., Karakas, A. I., et al. 2004, ApJ, 615, 934

Magrini, L., Vescovi, D., Casali, G., et al. 2021, A\&A, 646, L2

Marigo, P. 2002, A\&A, 387, 507

Marigo, P., \& Aringer, B. 2009, A\&A, 508, 1539

Meynet, G., \& Arnould, M. 2000, A\&A, 355, 176

Nucci, M. C., \& Busso, M. 2014, ApJ, 787, 141

Olive, K. A., \& Vangioni, E. 2019, MNRAS, 490, 4307

Palmerini, S., Trippella, O., Busso, M., et al. 2018, Geochim. Cosmochim. Acta, 221,21

Piersanti, L., Cristallo, S., \& Straniero, O. 2013, ApJ, 774, 98

Prantzos, N., Abia, C., Limongi, M., Chieffi, A., \& Cristallo, S. 2018, MNRAS, 476, 3432

Renda, A., Fenner, Y., Gibson, B. K., et al. 2004, MNRAS, 354, 575

Siess, L., Goriely, S., \& Langer, N. 2004, A\&A, 415, 1089

Spitoni, E., Matteucci, F., Jönsson, H., Ryde, N., \& Romano, D. 2018, A\&A, 612, A16

Straniero, O., Gallino, R., \& Cristallo, S. 2006, Nucl. Phys. A, 777, 311

Trippella, O., \& La Cognata, M. 2017, ApJ, 837, 41

Trippella, O., Busso, M., Maiorca, E., Käppeler, F., \& Palmerini, S. 2014, ApJ, 787,41

Trippella, O., Busso, M., Palmerini, S., Maiorca, E., \& Nucci, M. C. 2016, ApJ, 818,125

Ventura, P., \& Marigo, P. 2010, MNRAS, 408, 2476

Vescovi, D., Piersanti, L., Cristallo, S., et al. 2019, A\&A, 623, A126

Vescovi, D., Cristallo, S., Busso, M., \& Liu, N. 2020, ApJ, 897, L25

Vlemmings, W. 2019, IAU Symp., 343, 19

Wallner, A., Bichler, M., Buczak, K., et al. 2016, Phys. Rev. C, 93, 045803

Woosley, S. E., \& Haxton, W. C. 1988, Nature, 334, 45 